The Astrophysical Journal, 671:689-694, 2007 December 10

(C) 2007. The American Astronomical Society. All rights reserved. Printed in U.S.A.

\title{
THE RADIO EVOLUTION OF SN 2001gd
}

\author{
Christopher J. Stockdale \\ Physics Department, Marquette University, P.O. Box 1881, Milwaukee, WI 53214-1881; christopher.stockdale@mu.edu \\ Christopher L. WiLLIAMs ${ }^{1}$ \\ Naval Research Laboratory, Code 7213, Washington, DC 20375-5351; clmw@mit.edu \\ Kurt W. WeILER \\ Naval Research Laboratory, Code 7210, Washington, DC 20375-5320; kurt.weiler@nrl.navy.mil \\ Nino Panagia 2,3 \\ Space Telescope Science Institute, 3700 San Martin Drive, Baltimore, MD 21218; panagia@stsci.edu \\ Richard A. SRAmeK \\ National Radio Astronomy Observatory, P.O. Box O, Socorro, NM 87801; dsramek@nrao.edu \\ SCHUYLER D. VAN DYK \\ IPAC, Mail Code 100-22, California Institute of Technology, Pasadena, CA 91125; vandyk@ipac.caltech.edu \\ AND \\ Matthew T. Kelley \\ Physics Department, Marquette University, P.O. Box 1881, Milwaukee, WI 53214-1881; matthew.kelley@mu.edu \\ Received 2007 July 8; accepted 2007 August 7
}

\begin{abstract}
We present the results of observations of the radio emission from SN 2001gd in NGC5033 from 2002 February 8 through 2006 September 25. The data were obtained using the VLA at wavelengths of $1.3 \mathrm{~cm}(22.4 \mathrm{GHz}), 2 \mathrm{~cm}$ $(14.9 \mathrm{GHz}), 3.6 \mathrm{~cm}(8.4 \mathrm{GHz}), 6 \mathrm{~cm}(4.9 \mathrm{GHz})$, and $20 \mathrm{~cm}(1.5 \mathrm{GHz})$, with one upper limit at $90 \mathrm{~cm}(0.3 \mathrm{GHz}) . \mathrm{In}$ addition, one detection has been provided by the GMRT at $21 \mathrm{~cm}(1.4 \mathrm{GHz})$. SN $2001 \mathrm{gd}$ was discovered in the optical well past maximum light, so that it was not possible to obtain many of the early radio "turn-on" measurements that are important for estimating the local CSM properties. Only at $20 \mathrm{~cm}$ were turn-on data available. However, our analysis and fitting of the radio light curves, along with the assumption that the Type IIb SN 2001gd resembles the much better studied Type IIb SN 1993J, enables us to describe the radio evolution as being very regular through day $~ 550$ and consistent with a nonthermal-emitting model with a thermal absorbing CSM. The presence of SSA at early times is implied by the data, but determination of the exact relationship between the SSA component from the emitting region and the free-free absorption component from the CSM is not possible, as there are insufficient early measurements to distinguish between models. After day $\sim 550$, the radio emission exhibits a dramatically steeper decline rate, which, assuming similarity to SN 1993J, can be described as an exponential decrease with an $e$-folding time of 500 days. We interpret this abrupt change in the radio flux density decline rate as implying a transition of the shock front into a more tenuous region of circumstellar material. A similar change in radio evolution has been seen earlier in other SNe, such as SN 1988Z, SN 1980K, and SN 1993J.
\end{abstract}

Subject headings: galaxies: individual (NGC 5033) — radio continuum: stars — supernovae: individual (SN 2001gd)

\section{INTRODUCTION}

Supernova (SN) 2001gd was discovered at magnitude 16.5 on 2001 November 24.820 UT by Nakano et al. (2001) located $\sim 3^{\prime}$ north-northwest of the center of NGC 5033. Nakano et al. (2001) report a position of $\alpha=13^{\mathrm{h}} 13^{\mathrm{m}} 23.89^{\mathrm{s}}, \delta=+36^{\circ} 38^{\prime} 17.7^{\prime \prime}$ (J2000.0), for the SN and note that nothing was detected at this position in previous CCD images taken 179 days earlier (2001 May 29) to a limiting magnitude of 19. Ten days later, Matheson et al. (2001) obtained a spectrum of the SN, identifying it as a Type IIb SN well past its maximum light, with weak helium lines and nebular-phase lines of magnesium, oxygen, and calcium

\footnotetext{
1 Current address: Kavli Institute for Astrophysics and Space Research, Massachusetts Institute of Technology, Cambridge, MA 02139.

2 INAF-Osservatorio Astrofisico di Catania, Via S. Sofia 78, I-95128 Catania, Italy.

3 Supernova Ltd., Olde Yard Village 131, Northsound Road, Virgin Gorda, British Virgin Islands.
}

beginning to dominate. They noted that this spectrum was nearly identical to that of Type IIb SN 1993J on day 93 after its explosion. Using this suggestion to constrain the explosion date for the $\mathrm{SN}$, we assume that the optical discovery occurred $\sim 83$ days after the explosion, and therefore for calculating the age of the supernova we assume an explosion date of 2001 September 3, which is the same explosion date assumed by Pérez-Torres et al. (2005).

Tully (1988) gives the distance to NGC 5033 as $18.7 \mathrm{Mpc}$ (calculated with $H_{0}=75 \mathrm{~km} \mathrm{~s}^{-1} \mathrm{Mpc}^{-1}$ ). Accepting their distance calculation and correcting it with an updated value of the Hubble constant $H_{0}=65 \mathrm{~km} \mathrm{~s}^{-1} \mathrm{Mpc}^{-1}$ gives a distance to the $\mathrm{SN}$ of $\sim 21.6 \mathrm{Mpc}$. We note, however, that NGC 5033 is the host of several historical supernovae, including the radio-emitting SN 1985L (Van Dyk et al. 1998). If we follow Van Dyk et al. (1998) and estimate the Tully (1988) distance with Hubble constant $H_{0}=70 \mathrm{~km} \mathrm{~s}^{-1} \mathrm{Mpc}^{-1}$, we obtain a distance to NGC 5033 of $\sim 20 \mathrm{Mpc}$. Since a change in the distance estimate of $<10 \%$ 
TABLE 1

Flux Density Measurements for SN 2001gd $\mathrm{g}^{\mathrm{a}}$

\begin{tabular}{|c|c|c|c|c|c|c|c|c|}
\hline $\begin{array}{l}\text { Obs. Date } \\
\text { (UT) }\end{array}$ & $\begin{array}{c}\text { Age } \\
\text { (days) }\end{array}$ & Telescope & $\begin{array}{c}S(90 \mathrm{~cm}) \\
(\mathrm{mJy})\end{array}$ & $\begin{array}{c}S(20 \mathrm{~cm}) \\
(\mathrm{mJy})\end{array}$ & $\begin{array}{c}S(6 \mathrm{~cm}) \\
(\mathrm{mJy})\end{array}$ & $\begin{array}{c}S(3.6 \mathrm{~cm}) \\
(\mathrm{mJy})\end{array}$ & $\begin{array}{c}S(2 \mathrm{~cm}) \\
(\mathrm{mJy})\end{array}$ & $\begin{array}{c}S(1.3 \mathrm{~cm}) \\
(\mathrm{mJy})\end{array}$ \\
\hline 2001 Sep $3 .$. & $\equiv 0$ & & & & & & & \\
\hline 2002 Feb $8 \ldots \ldots \ldots \ldots \ldots \ldots$ & 159 & VLA-A & $\ldots$ & $0.85 \pm 0.15$ & $5.05 \pm 0.26$ & $4.09 \pm 0.21$ & $\ldots$ & $1.37 \pm 0.18$ \\
\hline 2002 Mar 2 .................... & 180 & VLA-A & $\ldots$ & $1.41 \pm 0.20$ & $5.41 \pm 0.28$ & $3.73 \pm 0.20$ & $2.18 \pm 0.24$ & $1.34 \pm 0.18$ \\
\hline 2002 Mar $14 \ldots \ldots \ldots \ldots \ldots \ldots$ & 192 & VLA-A & $\ldots$ & $1.84 \pm 0.37$ & $4.46 \pm 0.24$ & $\ldots$ & $\ldots$ & $\ldots$ \\
\hline 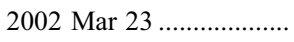 & 201 & VLA-A & $\ldots$ & $1.83 \pm 0.36$ & $\ldots$ & $3.66 \pm 0.21$ & $2.33 \pm 0.32$ & $1.00 \pm 0.19$ \\
\hline 2002 Apr $6 \ldots \ldots \ldots \ldots \ldots \ldots \ldots \ldots \ldots \ldots \ldots \ldots$ & 215 & VLA-A & $\ldots$ & $1.99 \pm 0.37$ & $5.33 \pm 0.28$ & $3.78 \pm 0.21$ & $2.66 \pm 0.30$ & $\ldots$ \\
\hline 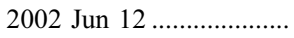 & 282 & VLA-B & $\ldots$ & $3.23 \pm 0.37$ & $4.84 \pm 0.25$ & $3.10 \pm 0.17$ & $\ldots$ & $\ldots$ \\
\hline 2002 Jun $26^{\mathrm{b}} \ldots \ldots \ldots \ldots \ldots \ldots$ & 296 & VLBA & $\ldots$ & $\ldots$ & $\ldots$ & $3.83 \pm 0.19$ & $\ldots$ & $\ldots$ \\
\hline 2002 Jul $16 \ldots \ldots \ldots \ldots \ldots \ldots$ & 317 & VLA-B & $\ldots$ & $2.73 \pm 0.31$ & $4.26 \pm 0.23$ & $2.39 \pm 0.14$ & $\ldots$ & $\ldots$ \\
\hline 2002 Sep $22^{\mathrm{c}} \ldots \ldots \ldots \ldots \ldots \ldots$ & 384 & GMRT & $\ldots$ & $3.55 \pm 0.22$ & $\ldots$ & $\ldots$ & $\ldots$ & $\ldots$ \\
\hline 2002 Oct $28 \ldots \ldots \ldots \ldots \ldots \ldots$ & 421 & VLA-C & $\ldots$ & $\ldots$ & $\ldots$ & $2.07 \pm 0.14$ & $0.89 \pm 0.18$ & $0.81 \pm 0.16$ \\
\hline 2002 Nov $8 \ldots \ldots \ldots \ldots \ldots \ldots$ & 432 & VLA-C & $\ldots$ & $4.22 \pm 0.48$ & $3.26 \pm 0.20$ & $\ldots$ & $\ldots$ & $\ldots$ \\
\hline 2003 Apr $8^{\mathrm{b}} \ldots \ldots \ldots \ldots \ldots \ldots$ & 582 & VLA-D & $\ldots$ & $3.89 \pm 0.40$ & $1.85 \pm 0.24$ & $1.02 \pm 0.05$ & $0.63 \pm 0.13$ & $0.39 \pm 0.12$ \\
\hline 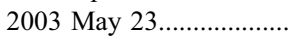 & 627 & VLA-A & $<3.09$ & $\ldots$ & $\ldots$ & $0.96 \pm 0.10$ & $\ldots$ & $<0.39$ \\
\hline 2003 May $26 \ldots \ldots \ldots \ldots \ldots \ldots$ & 630 & VLA-A & $\ldots$ & $3.47 \pm 0.45$ & $1.67 \pm 0.10$ & $\ldots$ & $\ldots$ & $\ldots$ \\
\hline 2004 Sep $10 \ldots \ldots \ldots \ldots \ldots \ldots$ & 1104 & VLA-A & $\ldots$ & $1.04 \pm 0.14$ & $\ldots$ & $<0.18$ & $<0.79$ & $<0.79$ \\
\hline 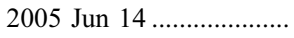 & 1380 & VLA-C & $\ldots$ & $<1.12$ & $<0.21$ & $<0.14$ & $<0.48$ & $<0.42$ \\
\hline 2006 Jan $24 \ldots \ldots \ldots \ldots \ldots \ldots$ & 1605 & VLA-D & $\ldots$ & $\ldots$ & $<0.39$ & $<0.6$ & $\ldots$ & $\ldots$ \\
\hline 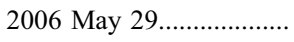 & 1729 & VLA-BnA & $\ldots$ & $<0.32$ & $\ldots$ & $\ldots$ & $\ldots$ & $\ldots$ \\
\hline 2006 Sep $25 \ldots \ldots \ldots \ldots \ldots \ldots$ & 1849 & VLA-B & $\ldots$ & $\ldots$ & $<0.29$ & $\ldots$ & $\ldots$ & $\ldots$ \\
\hline
\end{tabular}

a All upper limits are $3 \sigma$.

b From Pérez-Torres et al. (2005); Pérez-Torres et al. (2005) also measured a $3 \sigma$ upper limit of $<0.3 \mathrm{mJy}$ for SN $2001 \mathrm{gd}$ on $2003 \mathrm{April} 8$ at $0.7 \mathrm{~cm}$ (43.3 GHz).

c Chandra et al. (2002).

will not significantly affect any of our estimates of the SN physical properties, in this paper, for consistency with the previous radio work, we use the Van Dyk distance of $20 \mathrm{Mpc}$. However, it should be noted that Pérez-Torres et al. (2005) assumed a much shorter distance of $13.1 \mathrm{Mpc}$.

SN 2001gd was first detected at radio wavelengths on 2002 February 8.54 by Stockdale et al. (2002) using the Very Large Array (VLA) ${ }^{4}$ at $1.3 \mathrm{~cm}(22.4 \mathrm{GHz}), 3.6 \mathrm{~cm}(8.4 \mathrm{GHz}), 6 \mathrm{~cm}$ $(4.9 \mathrm{GHz})$, and $20 \mathrm{~cm}(1.5 \mathrm{GHz})$. They report a radio position of $\alpha=13^{\mathrm{h}} 13^{\mathrm{m}} 23.889^{\mathrm{s}}, \delta=+36^{\circ} 38^{\prime} 18.14^{\prime \prime}(\mathrm{J} 2000.0)$ with uncertainties of $\pm 0.2^{\prime \prime}$ in each coordinate, which agrees with the reported optical position of Nakano et al. (2001) to within the errors.

Stockdale et al. (2003) presented the results of initial radio monitoring of SN 2001gd between 2002 February 8 and October 28 . Their results showed that the radio emission evolves very smoothly, consistent with the nonthermal-emitting, thermalabsorbing model described by Weiler et al. (2002). Their results also indicate a circumstellar medium (CSM) with significant structure embedded in a uniform medium, similar to that proposed for Type IIb SN 1993J (Van Dyk et al. 1994; Weiler et al. 2007). The similar optical spectra and early radio evolution of both SN 1993J and SN 2001gd indicate that the two Type IIb SNe may have a number of similarities.

Pérez-Torres et al. (2005) conducted VLBI observations of SN 2001gd at $3.6 \mathrm{~cm}(8.4 \mathrm{GHz})$ on 2002 June 26 and 2003 April 8. Although they were unable to resolve any structure in the $\mathrm{SN}$, they were able to estimate the $\mathrm{SN}$ angular size through interferometer visibility fitting and found that their data suggested a possible deceleration in the expansion (although their data were not conclusive). Using our assumed distance of $20 \mathrm{Mpc}$ to the host galaxy and the angular size limits estimated by Pérez-Torres

\footnotetext{
${ }^{4}$ The VLA is operated by the National Radio Astronomy Observatory, which is a facility of the National Science Foundation, operated under cooperative agreement by Associated Universities, Inc.
}

et al. (2005), we calculate the upper limit to the expansion velocity of the blast wave to be $\$ 22,000 \mathrm{~km} \mathrm{~s}^{-1}$ for the 2002 June 26 VLBI observation (day 296). Depending on the exact nature of the emitting region, the upper limit may be as low as $\lesssim 19,000 \mathrm{~km} \mathrm{~s}^{-1}$. Thus, for the purposes of this paper, and for comparison with SN 1993J, we assume an expansion velocity of $15,000 \mathrm{~km} \mathrm{~s}^{-1}$.

Pérez-Torres et al. (2005) conducted multifrequency radio observations with the VLA on 2003 April 8 to complement their VLBI data, obtaining a spectrum consistent with synchrotron emission partially suppressed by free-free absorption. They also obtained X-ray data on SN 2001gd from XMM-Newton observations of NGC5033 on 2001 July 2 and 2002 December 18 and report the detection of X-ray emission on the latter date consistent with the expected reverse shock emission from typical Type II SNe.

Recent VLA observations indicate a dramatic change in the evolution of the radio emission from SN $2001 \mathrm{gd}$. These new observations and their implications are presented in this paper.

\section{RADIO OBSERVATIONS}

New radio observations of SN $2001 \mathrm{gd}$ have been made with the VLA at $1.3 \mathrm{~cm}(22.4 \mathrm{GHz}), 2 \mathrm{~cm}(14.9 \mathrm{GHz}), 3.6 \mathrm{~cm}(8.4 \mathrm{GHz})$, $6 \mathrm{~cm}(4.9 \mathrm{GHz})$, and $20 \mathrm{~cm}(1.5 \mathrm{GHz})$, with one upper limit at $90 \mathrm{~cm}(0.3 \mathrm{GHz})$ from 2002 November 8 through 2006 September 25 and are presented in Table 1 and Figure 1. For completeness and ease of reference, we include the previously published results from Stockdale et al. (2003) and Pérez-Torres et al. (2005) as well as one measurement from the Giant Metrewave Radio Telescope (GMRT; Chandra et al. 2002) in both Table 1 and Figure 1.

The techniques of observation, editing, calibration, and error estimation are described in previous publications on the radio emission from SNe (see, e.g., Weiler et al. 1986, 2002; Sramek \& Weiler 2003). We used 3C 286 as the primary calibrator for flux density bootstrapping, while $\mathrm{J} 1310+323$, with a defined position of $\alpha=13^{\mathrm{h}} 10^{\mathrm{m}} 28.66^{\mathrm{s}}, \delta=+32^{\circ} 20^{\prime} 43.8^{\prime \prime}$ served as the phase calibrator and secondary flux density calibrator. The flux densities 


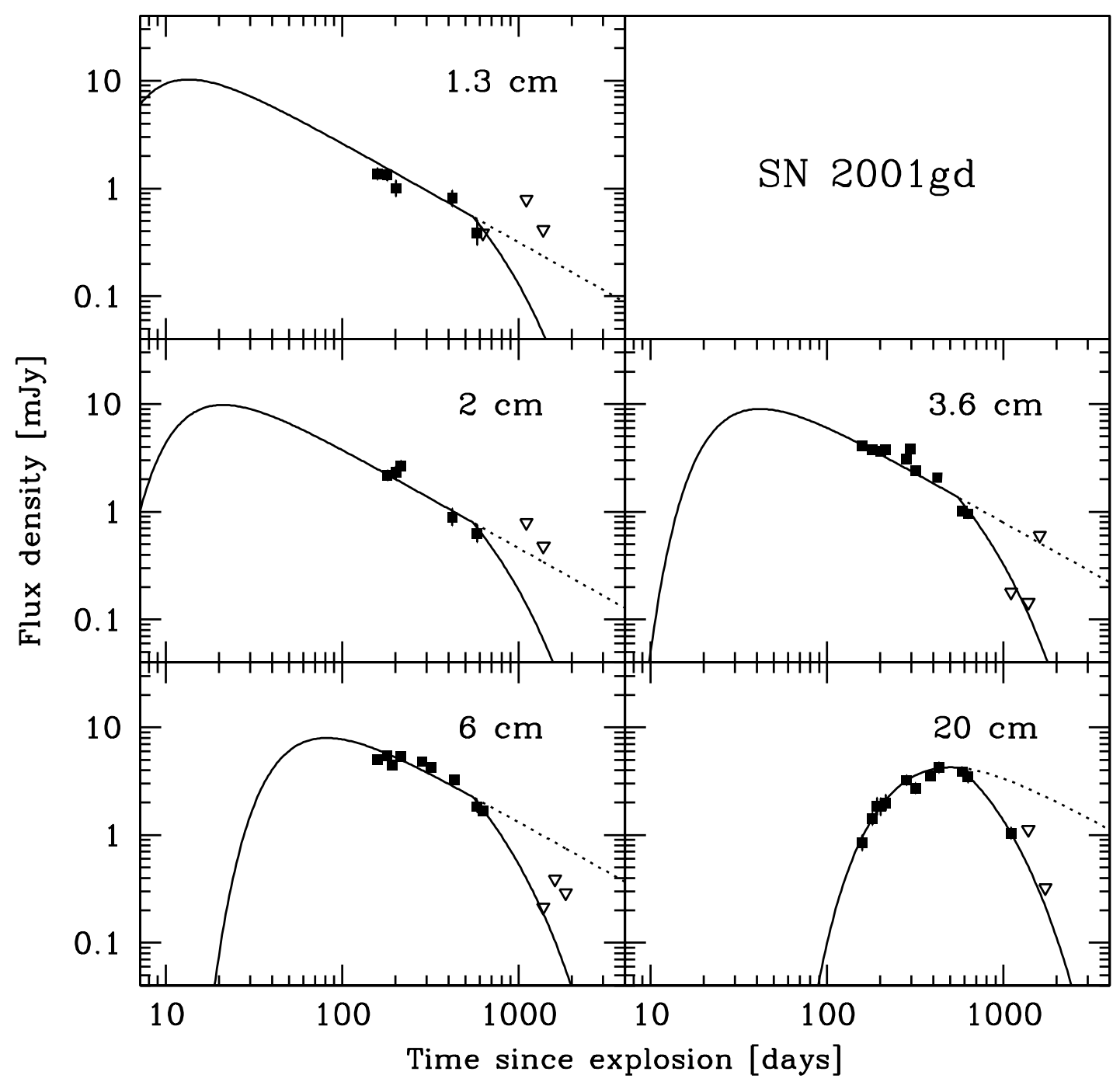

FIG. 1.- Radio light curves for SN 2001 gd in NGC 5033. The five wavelengths, $1.3 \mathrm{~cm}$ (22.4 GHz; top left), $2 \mathrm{~cm}(14.9 \mathrm{GHz}$; middle left), $3.6 \mathrm{~cm}$ ( $8.4 \mathrm{GHz}$; middle right), $6 \mathrm{~cm}(4.9 \mathrm{GHz}$; bottom left $)$, and $20 \mathrm{~cm}(1.5 \mathrm{GHz}$; bottom right), are shown together with their best-fit model light curves. The $\mathrm{SN}$ age is in days since the adopted explosion date of 2001 September 3. Because the decline index $\beta$ of the radio emission steepened after day 550, following the example of SN 1993J (Weiler et al. 2007), we have fitted the late data after day 550 with an exponential decline with an $e$-folding time of 500 days. The inverted open triangles represent $3 \sigma$ upper limits and the dotted lines show the extrapolation of the fit to the early data before day $\sim 550$ given in Table 3 .

of $\mathrm{J} 1310+323$ are shown in Table 2 and plotted in Figure 2. Like most phase calibrators, $\mathrm{J} 1310+323$ shows some variation in its flux density because phase calibrators are selected for their proximity to the $\mathrm{SN}$ and unresolvable angular size rather than their flux stability. The primary calibrator is therefore used to determine the absolute flux density of the secondary calibrator at each epoch of the observations.

The listed flux density measurement errors are determined based on both the intrinsic rms background noise level of the particular radio map and a fractional error, $\epsilon$, proportional to the $\mathrm{SN}$ flux. The rms map noise results from both small unresolved fluctuations in the background emission and random map fluctuations due to receiver noise, while the fractional error, $\epsilon$, accounts for the inaccuracy of VLA flux density calibration (see, e.g., Weiler et al. 1986) and possible deviations of the primary calibrator from an absolute flux density scale. The final errors $\left(\sigma_{f}\right)$ given for the measurements of SN 2001gd are taken as the addition in quadrature of these components:

$$
\sigma_{f}^{2}=\left(\epsilon S_{0}\right)^{2}+\sigma_{0}^{2},
$$

where $S_{0}$ is the measured flux density, $\sigma_{0}$ is the map rms background noise for each observation, and $\epsilon=0.15$ for $90 \mathrm{~cm}$, $\epsilon=0.1$ for $20 \mathrm{~cm}, \epsilon=0.05$ for $6 \mathrm{~cm}$ and $3.6 \mathrm{~cm}, \epsilon=0.075$ for $2 \mathrm{~cm}$, and $\epsilon=0.1$ for $1.3 \mathrm{~cm}$.

\section{PARAMETERIZED RADIO LIGHT CURVES}

From the most recent RSN modeling discussion of Weiler et al. (2002) and Sramek \& Weiler (2003) we use a parameterized model:

$$
\begin{aligned}
S(\mathrm{mJy})= & K_{1}\left(\frac{\nu}{5 \mathrm{GHz}}\right)^{\alpha}\left(\frac{t-t_{0}}{1 \text { day }}\right)^{\beta} e^{-\tau_{\text {external }}} \\
& \times\left(\frac{1-e^{-\tau_{\mathrm{CSM}_{\text {clumps }}}}}{\tau_{\mathrm{CSM}_{\text {clumps }}}}\right)\left(\frac{1-e^{-\tau_{\text {internal }}}}{\tau_{\text {internal }}}\right),
\end{aligned}
$$

with

$$
\tau_{\text {external }}=\tau_{\mathrm{CSM}_{\text {homogeneous }}}+\tau_{\text {distant }}
$$


TABLE 2

Flux Density Measurements for Secondary Calibrator J1310+323

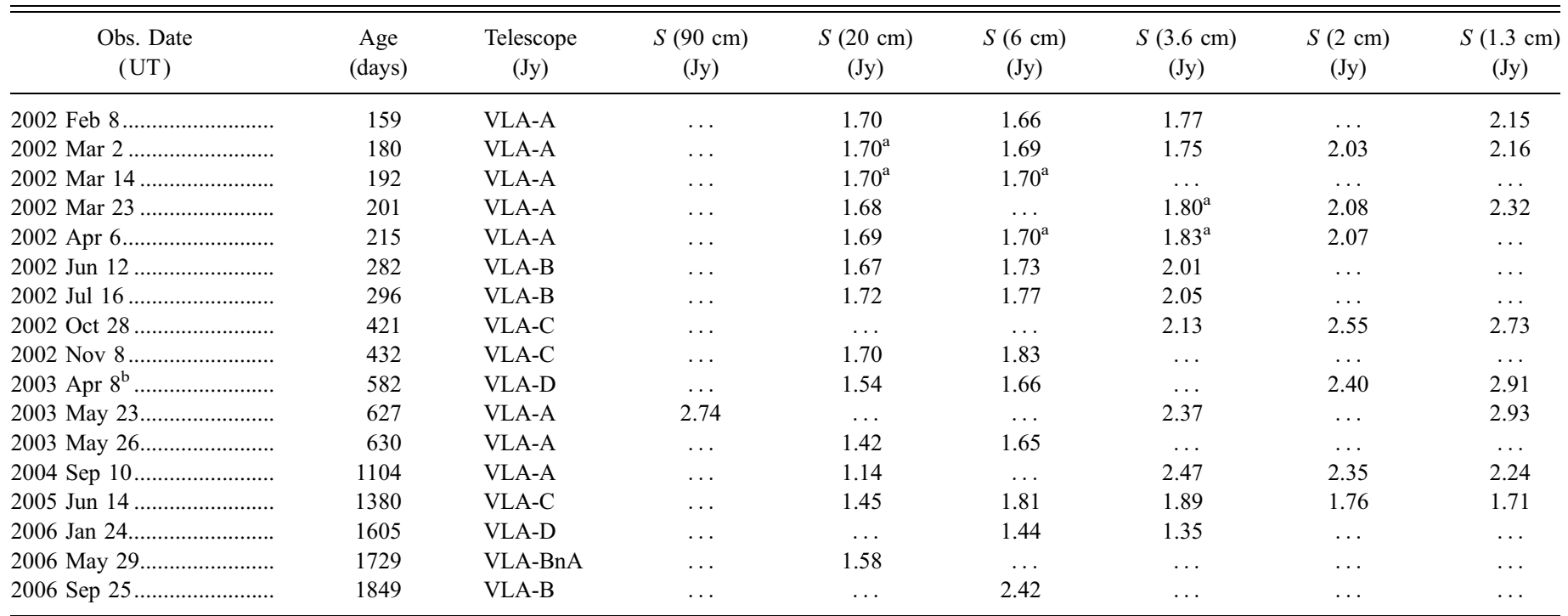

a Value was not measured but was interpolated from the closest calibration at that frequency.

b From Pérez-Torres et al. (2005); Pérez-Torres et al. (2005) also measured a value of 2.58 Jy for J1310+323 on $2003 \mathrm{Apr} 08$ at $0.7 \mathrm{~cm}$ (43.3 GHz).

where

$$
\begin{gathered}
\tau_{\mathrm{CSM}_{\text {homogeneous }}}=K_{2}\left(\frac{\nu}{5 \mathrm{GHz}}\right)^{-2.1}\left(\frac{t-t_{0}}{1 \text { day }}\right)^{\delta}, \\
\tau_{\text {distant }}=K_{4}\left(\frac{\nu}{5 \mathrm{GHz}}\right)^{-2.1},
\end{gathered}
$$

and

$$
\tau_{\mathrm{CSM}_{\mathrm{clumps}}}=K_{3}\left(\frac{\nu}{5 \mathrm{GHz}}\right)^{-2.1}\left(\frac{t-t_{0}}{1 \mathrm{day}}\right)^{\delta^{\prime}}
$$

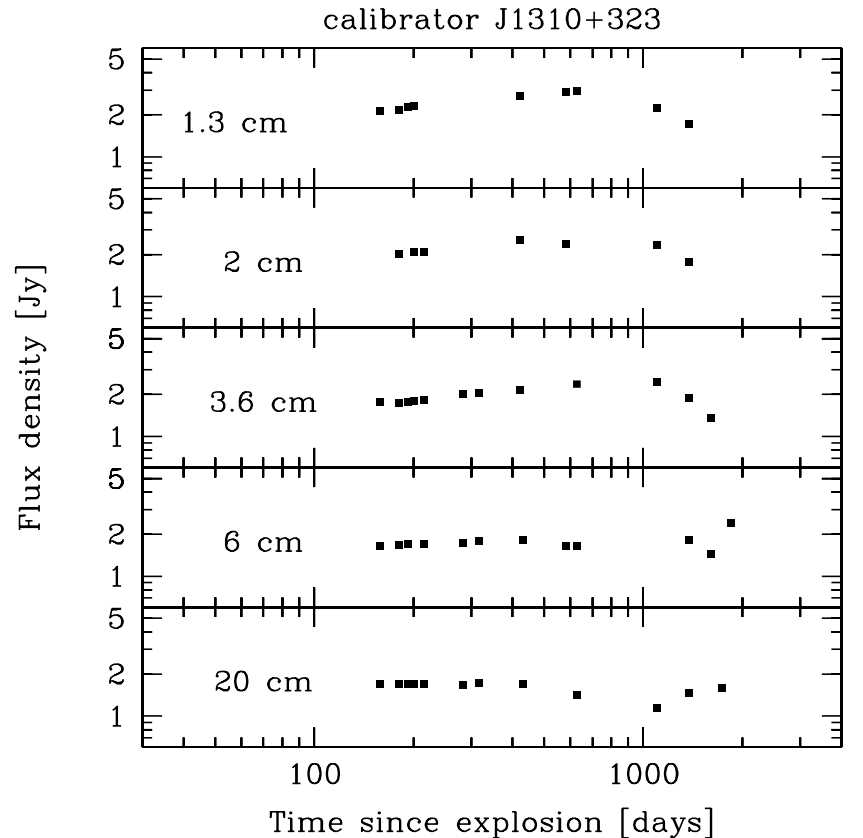

FIG. 2.-Flux density measurements of the secondary calibrator J1310+323 at the five wavelengths of $1.3 \mathrm{~cm}(22.4 \mathrm{GHz}$; top panel $), 2 \mathrm{~cm}(14.9 \mathrm{GHz}$; one below top $), 3.6 \mathrm{~cm}(8.4 \mathrm{GHz}$; middle $), 6 \mathrm{~cm}(4.9 \mathrm{GHz}$; one above bottom $)$, and $20 \mathrm{~cm}(1.5 \mathrm{GHz}$; bottom $)$. with $K_{1}, K_{2}, K_{3}$, and $K_{4}$ determined from fits to the data and corresponding to the flux density $\left(K_{1}\right)$, homogeneous $\left(K_{2}, K_{4}\right)$, and clumpy or filamentary $\left(K_{3}\right)$ free-free absorption (FFA) at $5 \mathrm{GHz}$, formally but not necessarily physically, one day after the explosion date $t_{0}$. For a more detailed explanation of the physical meaning of the parameters, the reader should consult the references cited above.

Since it is physically realistic and may be needed in some RSNe from which radio observations have been obtained at early times and high frequencies, equation (2) also includes the possibility of an internal absorption term $\left(\tau_{\text {internal }}\right)$, which may consist of two parts: synchrotron self-absorption (SSA; $\left.\tau_{\text {internal }}{ }_{\text {SSA }}\right)$ and mixed, thermal FFA/nonthermal emission $\left(\tau_{\text {internal }_{\mathrm{FFA}}}\right)$ :

$$
\begin{gathered}
\tau_{\text {internal }}=\tau_{\text {internal }_{\mathrm{SSA}}}+\tau_{\text {internal }_{\mathrm{FFA}}}, \\
\tau_{\text {internal }_{\mathrm{SSA}}}=K_{5}\left(\frac{\nu}{5 \mathrm{GHz}}\right)^{\alpha-2.5}\left(\frac{t-t_{0}}{1 \text { day }}\right)^{\delta^{\prime \prime}}, \\
\tau_{\text {internal }_{\mathrm{FFA}}}=K_{6}\left(\frac{\nu}{5 \mathrm{GHz}}\right)^{-2.1}\left(\frac{t-t_{0}}{1 \text { day }}\right)^{\delta^{\prime \prime \prime}},
\end{gathered}
$$

with $K_{5}$ corresponding to the internal, nonthermal $\left(\nu^{\alpha-2.5}\right)$ SSA and $K_{6}$ corresponding to the internal thermal $\left(\nu^{-2.1}\right)$ free-free absorption mixed with nonthermal emission, at $5 \mathrm{GHz}$, formally but not necessarily physically, one day after the explosion date $t_{0}$. The parameters $\delta^{\prime \prime}$ and $\delta^{\prime \prime \prime}$ describe the time dependence of the optical depths for the SSA and FFA internal absorption components, respectively.

Application of this basic parameterization has been shown to be effective in describing the physical characteristics of the presupernova system, its CSM, and its final stages of evolution before explosion for objects ranging from the two decades of monitoring the complex radio emission from SN 1979C (Montes et al. 2000) through the unusual SN 1998bw (GRB 980425; Weiler et al. 2001) to the very well studied SN 1993J (Weiler et al. 2007).

\section{RADIO LIGHT-CURVE DESCRIPTION}

The multifrequency radio data for SN 2001gd are listed in Table 1 and displayed in Figure 1. Examination of the data indicates 
that radio evolution of the $\mathrm{SN}$ is best described by two distinct time intervals: an "early" interval before day 550 and a "late" interval thereafter. Although the precise date of the observed "break" is not well defined, it appears that around the time of the observations of Pérez-Torres et al. (2005) on 2003 April 8 (day 582) the rate of flux decline markedly steepened, such that the data can no longer be adequately described by the model appropriate for earlier times. Similar "breaks" have been observed in the radio light curves of other RSNe, including SN $1988 Z$ (Williams et al. 2002), SN 1980K (Montes et al. 1998), and SN 1993J (Weiler et al. 2007).

An extensive search for the best parameter fits to equations $(2)-(9)$ was carried out on the early interval (day $<550)$ by minimizing the reduced $\chi^{2}\left(\chi_{\text {red }}^{2}\right)$. Since there are only four radio detections after the measurements on day 582, the "late" radio light-curve behavior is very poorly defined, although it can be noted that the few detections and the several upper limits indicate that the decline rate has significantly steepened and, following the example of SN 1993J (Weiler et al. 2007), are consistent with an exponential decline with an $e$-folding time of 500 days.

The early-epoch fitting parameters are consistent with a homogeneous $\left(K_{2}\right)$ CSM, although more complex structures, such as a CSM containing filamentary $\left(K_{3}\right)$ components, cannot be ruled out. No evidence is seen for any distant, homogeneous thermal absorption $\left(K_{4}=0\right)$, and the very early data $(<100$ days $)$ are too sparse to determine from the fitting if SSA $\left(K_{5}\right)$, or mixed, internal, freefree absorption/nonthermal emission $\left(K_{6}\right)$ may have been present.

However, we have estimated the brightness temperatures for the early (before day 550) $20 \mathrm{~cm}$ emission based on a pure FFA model and found that they exceed the limiting temperature for synchrotron emission of $\sim 10^{11.5} \mathrm{~K}$ calculated by Readhead (1994), which was also the case for SN 1993J (Weiler et al. 2007). Given our inability to model this early absorption based purely on our sparse early data, we have chosen to assume that SN 2001gd has a similar brightness temperature evolution $\left(\sim 10^{11} \mathrm{~K}\right.$ at peak $)$ for the $20 \mathrm{~cm}$ emission as it becomes optically thin. Given that SN 2001gd and SN 1993J have similar characteristics at early times, we estimate, as was the case for SN 1993J, that a significant SSA component is required for SN 2001gd along with the FFA component determined from fitting that describes the rapid rise of the $20 \mathrm{~cm}$ data well. The best parameters that we can estimate for the early epoch of SN $2001 \mathrm{gd}$ with these assumptions are given in Table 3 . We note an apparent similarity between the $\delta$ and $\delta^{\prime \prime}$ terms from our modeling of the SN 2001gd radio emission. This is similar to the case of SN 1993J for which the two values were $\delta=-1.88$ and $\delta^{\prime \prime}=-2.05$. However, it must be stated that, from pure modeling, our sparse early data only constrain the values of $\delta$ and $\delta^{\prime \prime}$ for SN 2001gd to lie between -1 and -2 . To narrow the range of these values, we have therefore assumed a value of $\delta=-1.88$ to match the value for $\delta$ determined for SN 1993J (Weiler et al. 2007).

The late period appears to be optically thin at all observed frequencies, with a much steeper decline rate than is possible with the early data determined $\beta=-0.92$ (Fig. 1, dotted line). Again, if we assume a similarity to the exponential decline seen for SN 1993J (Weiler et al. 2007), the late epoch of SN 2001gd is consistent with an exponential decline after day 550, with an $e$-folding time of 500 days. This observed break in the radio light curves of SN $2001 \mathrm{gd}$ is interpreted as indicative of a rapid transition of the blast wave into a much more tenuous medium. This occurs much earlier for SN $2001 \mathrm{gd}$ (day 550) than that seen for SN 1993J (day 3100) by Weiler et al. (2007).

Before the break, SN 2001gd is still somewhat optically thick at $20 \mathrm{~cm}$, but after the break it appears to be optically thin. This
TABLE 3

Fitting Parameters for SN 2001gd

\begin{tabular}{|c|c|}
\hline Parameter & Early (day $<550$ ) \\
\hline$K_{1} \ldots \ldots \ldots \ldots \ldots \ldots \ldots \ldots \ldots \ldots \ldots \ldots \ldots$ & $7.5 \times 10^{2}$ \\
\hline 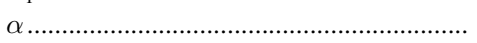 & -0.94 \\
\hline$\beta$ & -0.92 \\
\hline$K_{2}$ & $1.5 \times 10^{3}$ \\
\hline 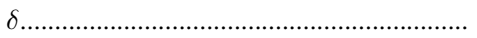 & -1.88 \\
\hline$K_{5}$ & $1.7 \times 10^{2}$ \\
\hline$\delta^{\prime \prime} \ldots \ldots$ & -1.5 \\
\hline$t_{0}^{\mathrm{a}}$ & 2001 Sep 3 \\
\hline 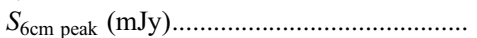 & 7.96 \\
\hline 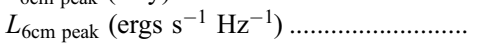 & $3.8 \times 10^{27}$ \\
\hline 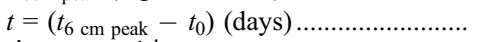 & 80 \\
\hline 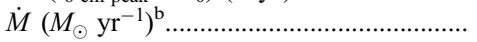 & $(0.7-5.6) \times 10^{-5}$ \\
\hline 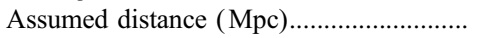 & 20 \\
\hline
\end{tabular}

discounts the possibility of the break being the result of a sudden change in the shock velocity or magnetic field properties at the shock front since such changes would not yield an optical depth reduction. Given the similar expansion velocities of $\lesssim 19,000 \mathrm{~km} \mathrm{~s}^{-1}$ for SN $2001 \mathrm{gd}$ (derived from Pérez-Torres et al. 2005; see $\S 1$ ) and 11,000-15,000 $\mathrm{km} \mathrm{s}^{-1}$ for SN 1993J (Marcaide et al. 1997; J. M. Marcaide et al. 2008, in preparation), it is clear that both supernovae had a period of enhanced mass-loss rate preceding their explosions. Thus, assuming a blast wave velocity for SN 2001 gd of 15,000 $\mathrm{km} \mathrm{s}^{-1}$ and a "standard" preexplosion wind velocity of $10 \mathrm{~km} \mathrm{~s}^{-1}$, this enhanced mass loss persisted for the $\sim 1800 \mathrm{yr}$ prior to the explosion. However, in the case of SN 1993J, this phase began $\sim 8800$ yr earlier than the explosion of its progenitor star. The shorter duration of the high presupernova mass-loss rate for SN $2001 \mathrm{gd}$ is also borne out in its faster rise and higher luminosity, indicating that there was less intervening CSM established prior to the $\mathrm{SN}$ explosion to contribute to the FFA at early epochs.

\subsection{The Mass-Loss Rate for the SN Progenitor}

Through use of this modeling, a number of physical properties of $\mathrm{SNe}$ can be determined from the radio observations. One of these is the mass-loss rate from the SN progenitor prior to explosion. From the Chevalier (1982a, 1982b) model, the turn-on of the radio emission for RSNe provides a measure of the ratio of presupernova mass-loss rate to wind velocity $\left(\dot{M} / w_{\text {wind }}\right)$ and Weiler et al. (1986) derived this ratio for the case of pure, thermal, external absorption by a homogeneous medium.

Given the limited amount of early radio data available for SN 2001gd, there is no need to invoke more complex models than the simple, uniform, external thermal absorption model, so that $\tau(t=1$ day $)=K_{2}$ and equation (16) of Weiler et al. (1986) can be written:

$$
\begin{aligned}
\frac{\dot{M}\left(M_{\odot} \mathrm{yr}^{-1}\right)}{\left(w_{\text {wind }} / 10 \mathrm{~km} \mathrm{~s}^{-1}\right)}= & 3.0 \times 10^{-6} \phi \tau_{\mathrm{CSM}_{\text {homogeneous }}^{0.5}} m^{-1.5} \\
& \times\left(\frac{v_{i}}{10^{4} \mathrm{~km} \mathrm{~s}^{-1}}\right)^{1.5}\left(\frac{t_{i}}{45 \text { days }}\right)^{1.5} \\
& \times\left(\frac{t}{t_{i}}\right)^{1.5 m}\left(\frac{T}{10^{4} \mathrm{~K}}\right)^{0.68}
\end{aligned}
$$


Here, the extra factor $\phi$ is a small correction that takes into account the fact that, for SN $2001 \mathrm{gd}$, the CSM density behaves like $\rho \propto r^{-1.61}$ (see below) instead of $r^{-2}$ as it would under the usual constant mass-loss rate assumption. The factor $\phi$ is given by the square root of the ratio of the integration constant for $\tau$ in the case of $\rho \propto r^{-1.61}$ to the one appropriate for $\rho \propto r^{-2}$, i.e.,

$$
\phi=\left(\frac{2 \times 1.61-1}{2 \times 2-1}\right)^{0.5}=0.86 .
$$

Since SN 2001gd is a Type IIb supernova, as was SN 1993J, and appears to be quite similar to it, we adopt for SN 2001gd some of the same parameters as those measured for SN 1993J. Thus, for SN 2001gd we assume $v_{i}=15,000 \mathrm{~km} \mathrm{~s}^{-1}$ at $t=45$ days, which is a value consistent with the results of Pérez-Torres et al. (2005), as discussed earlier.

We also adopt values of $T=20,000 \mathrm{~K}, w_{\text {wind }}=10 \mathrm{~km} \mathrm{~s}^{-1}$ (which is appropriate for a RSG wind), $t=\left(t_{6 \mathrm{~cm} \mathrm{peak}}-t_{0}\right)$ days from our best fits to the radio data, and $m=0.845$, as measured for SN $1993 \mathrm{~J}$ by J. M. Marcaide et al. (2008, in preparation). With the assumptions for the blast wave and CSM properties discussed above and the results for the best-fit parameters listed in Table 3, our estimated presupernova mass-loss rate is $\dot{M}=7.1 \times$ $10^{-6} M_{\odot} \mathrm{yr}^{-1}$ at 1 day before explosion.

The fitting parameter value of $\delta=-1.88$ indicates that the CSM density behaves like $\rho \propto r^{-1.61}$, so that the mass-loss rate for SN 2001gd was not constant but was decreasing in the years leading up to the explosion, i.e., $\dot{M} \propto r^{2} \rho w_{\text {wind }} \propto r^{0.39}$ for a constant $w_{\text {wind }}$.

Since the shock speed for SN $2001 \mathrm{gd}$ is assumed to be $\sim 15,000 \mathrm{~km} \mathrm{~s}^{-1}$ at $t=45$ days, but is variable with time $(v \propto$ $t^{-0.155}$ for $m=0.845$ from J. M. Marcaide et al. 2008, in preparation) and the presupernova wind velocity for an RSG is typically $\sim 10 \mathrm{~km} \mathrm{~s}^{-1}$, we calculate that the abrupt decline in the radio light curves starting around day $\sim 550$ implies that this change took place in the presupernova stellar wind $\sim 1800$ yr before explosion, when the mass-loss rate was as high as $5.6 \times 10^{-5} M_{\odot} \mathrm{yr}^{-1}$.
Integrating the mass-loss rate over the last $\sim 1800$ yr before explosion, we find that during that time, the progenitor star shed $\sim 0.07 M_{\odot}$ in a massive stellar wind. At earlier epochs of the progenitor's evolution the mass-loss rate was considerably lower, as indicated by the radio light-curve break discussed above and the transition of the shock wave to a lower density CSM at that time.

\section{CONCLUSIONS}

Figure 1 and Table 3 show that the radio light curves for SN 2001gd can be described by standard RSN models (Weiler et al. 1986, 1990, 2002; Montes et al. 1997) during its "early" evolution up to day $\sim 550$. After day $\sim 550$ the flux density decline rate clearly steepened, in comparison with SN 1993J (Weiler et al. 2007), to a form consistent with an exponential decay with $e$-folding time of 500 days. The "break" in the radio light curves for SN $2001 \mathrm{gd}$ is interpreted as being due to a change in the average CSM density. Similar breaks have been identified earlier in SN 1980K (Montes et al. 1998), SN 1988Z (Williams et al. 2002), and SN 1993J (Weiler et al. 2007).

Normal assumptions for presupernova stellar wind properties imply a dense wind with the mass-loss rate decreasing for the last $\sim 1800$ yr before explosion, from $\dot{M} \sim 5.6 \times 10^{-5}$ to $\dot{M} \sim 7.1 \times$ $10^{-6} M_{\odot} \mathrm{yr}^{-1}$ around the time of explosion. The mass-loss rate was sharply lower for times earlier than $\sim 1800 \mathrm{yr}$ before explosion.

K. W. W. wishes to thank the Office of Naval Research (ONR) for the 6.1 funding supporting his research. C. J. S. is a Cottrell Scholar of the Research Corporation, and work on this project has been supported by the NASA Wisconsin Space Grant Consortium. N. P. is Astronomer Emeritus at the Space Telescope Science Institute (STScI), which kindly provided research facilities and partial support for this work. Additional information and data on radio supernovae can be found at http://rsd-www.nrl.navy .mil/7213/weiler/sne-home.html and linked pages.
Chandra, P., Ray, A., \& Bhatnagar, S. 2002, IAU Circ. 7982, 2

Chevalier, R. A. 1982a, ApJ, 259, 302 1982b, ApJ, 259, L85

Marcaide, J. M., et al. 1997, ApJ, 486, L31

Matheson, T., Jha, S., Challis, P., Kirshner, R., \& Berlind, P. 2001, IAU Circ. 7765

Montes, M. J., van Dyk, S. D., Weiler, K. W., Sramek, R. A., \& Panagia, N. 1998, ApJ, 506, 874

Montes, M. J., Weiler, K. W., \& Panagia, N. 1997, ApJ, 488, 792

Montes, M. J., Weiler, K. W., Van Dyk, S. D., Panagia, N., Lacey, C. K., Sramek, R. A., \& Park, R. 2000, ApJ, 532, 1124

Nakano, S., Itagaki, K., Kushida, Y., Kushida, R., \& Dimai, A. 2001, IAU Circ. 7761

Pérez-Torres, M. A., et al. 2005, MNRAS, 360, 1055

Readhead, A. C. S. 1994, ApJ, 426, 51

Sramek, R. A., \& Weiler, K. W. 2003, Supernovae and Gamma-Ray Bursters (New York: Springer), 137

Stockdale, C. J., Weiler, K. W., Van Dyk, S. D., Montes, M. J., Panagia, N., Sramek, R. A., Perez-Torres, M. A., \& Marcaide, J. M. 2003, ApJ, 592, 900

\section{REFERENCES}

Stockdale, C. J., et al. 2002, IAU Circ. 7830

Tully, R. B. 1988, Nearby Galaxies Catalog (Cambridge: Cambridge Univ. Press) Van Dyk, S. D., Montes, M. J., Weiler, K. W., Sramek, R. A., \& Panagia, N. 1998, AJ, 115, 1103

Van Dyk, S., Weiler, K., Sramek, R., Rupen, M., \& Panagia, N. 1994, ApJ, 432, L115

Weiler, K. W., Panagia, N., \& Montes, M. 2001, ApJ, 562, 670

Weiler, K. W., Panagia, N., Montes, M. J., \& Sramek, R. A. 2002, ARA\&A, 40, 387

Weiler, K. W., Panagia, N., \& Sramek, R. A. 1990, ApJ, 364, 611

Weiler, K. W., Sramek, R. A., Panagia, N., van der Hulst, J. M., \& Salvati, M. 1986, ApJ, 301, 790

Weiler, K. W., Williams, C. L., Panagia, N., Stockdale, C. J., Kelley, M. T., Sramek, R. A., Van Dyk, S. D., \& Marcaide, J. M. 2007, ApJ, in press

Williams, C. L., Panagia, N., Van Dyk, S. D., Lacey, C. K., Weiler, K. W., \& Sramek, R. A. 2002, ApJ, 581, 396 